\title{
Idle peripheral intravenous cannulation: An observational cohort study of pre-hospital and emergency department practices
}

Hugo Evison ( $\square$ hugo.evison@gmail.com )

Queensland Ambulance Service https://orcid.org/0000-0003-2911-3335

\section{Amy Sweeny}

QLD Health

Jamie Ranse

Griffith University

Mercedes Carrington

QLD Health

\section{Nicole Marsh}

Royal Brisbane Hospital

Joshua Byrnes

Griffith University

Claire Rickard

Griffith University

\section{Peter Carr}

National University of Ireland Galway

Gerben Keijzers

QLD Health

Original research

Keywords: Cannula, emergency department, idle, pre-hospital, vascular access device.

Posted Date: November 13th, 2020

DOl: https://doi.org/10.21203/rs.3.rs-106404/v1

License: (c) (i) This work is licensed under a Creative Commons Attribution 4.0 International License.

Read Full License

Version of Record: A version of this preprint was published at Scandinavian Journal of Trauma, Resuscitation and Emergency Medicine on August 28th, 2021. See the published version at 
https://doi.org/10.1186/s13049-021-00941-y. 


\section{Abstract}

Background Unused ('idle') peripheral intravenous catheters (PIVC) are those not used within 24 hours of insertion. There is little data on cannulation practices and idle PIVC rates in emergency settings, especially the pre-hospital environment.

Methods This was an observational cohort study set in south-east Queensland, Australia using data from a large tertiary level emergency department (ED) and the local statutory ambulance service. Demographic, clinical and PIVC data were collected over two periods; 9 February - 18 March 2017 and 5 January - 4 February 2018. Adult patients were included if they were allocated an Australasian triage scale (ATS) category between 2 and 5, and had a PIVC inserted in the pre-hospital setting or ED. PIVC use was defined as idle if no fluids, medications or contrast were administered intravenously within 24 hours of insertion. Comparisons between pre-hospital and ED practice and idle PIVC status were undertaken using descriptive statistics and logistic regression.

Results A total of 1249 patients with a PIVC (372 pre-hospital; 877 ED) were included. Overall, 366 PIVCs (29.3\%; 95\% Cl 26.9\%-31.9\%) remained idle at 24h. In the pre-hospital group, 147 (39.5\%) PIVCs inserted were not used pre-hospital, and 74 (19.9\%) remained idle. In comparison, 292 (33.3\%) PIVCs placed in the ED remained idle. ED staff more frequently inserted PIVCs in the antecubital fossa than paramedics (65.5\% vs $49.7 \%)$, where forearm PIVC insertion was more common pre-hospital than in ED $(13.7 \%$ vs $7.4 \%)$. Nursing staff inserted idle PIVC at a rate of (35.1\%) compared to doctors (29.6\%) and paramedics $(19.9 \%)$. Having a PIVC insertion in the ED was the only factor significantly $(p=<.001)$ predicting an idle outcome (Odds Ratio: 2.4; 95\% Cl 1.7-3.3).

Conclusion One-third of PIVCs inserted within the emergency setting remained idle, suggesting unnecessary risk and costs. Pre-hospital and ED PIVC insertion practices differed, with idle PIVCs 2.4 times more prevalent if inserted in the ED than pre-hospital and with greater use of ante-cubital insertion. Reasons for these differences are not well understood and requires more targeted research.

\section{Background}

Establishing peripheral intravenous catheter (PIVC) access is a fundamental element for facilitating modern day emergency health care ${ }^{(1)}$. It allows for the administration of a variety of symptom-relieving and/or potentially lifesaving infusates ${ }^{(2)}$. The ubiquitous use and perceived low risk associated with this device has led to frequent and routine insertion ${ }^{(3)}$. However, PIVC insertion is described as a traumatic, invasive procedure, with over half of patients finding the procedure at least moderately painful( ${ }^{(4)}$. As $12-$ $25 \%$ of first time attempts are unsuccessful $(5,6)$, the possible distress caused by failure of the first attempt is an important consideration in the clinical decision making whether a PIVC should be inserted.

Inflammatory complications are common, and studies have reported that $18-54 \%$ of PIVCs are associated with phlebitis ${ }^{(6-10)}$. Serious infections can occur as the PIVC acts as a potential conduit for 
the introduction of infectious pathogens ${ }^{(11)}$. Previous research examining rates of intravascular related bloodstream infections (BSIs) found a pooled mean of $0.1 \%$ PIVC BSIs per 100 devices $^{(12)}$. Staphylococcus aureus bacteraemia carries the highest morbidity and mortality rate of healthcare associated infections and is associated with up to $25 \%$ of all PIVC related bloodstream infections ${ }^{(13,14)}$. The prolific use of the device implies many patients are suffering serious adverse events ${ }^{(15)}$.

A fundamental concept performing any procedure, including PIVC insertion, is to ensure potential benefits outweigh the risks. There are a wide range of clinical indications for PIVCs, making appropriate patient selection for insertion challenging. Although the clinical need for a PIVC is sometimes obvious, many are inserted in haemodynamically stable patients without a clear or immediate requirement. These PIVCs are often placed ' $j u s t$ in case, with negligible potential benefits, and studies report up to $50 \%$ remain idle and are never used $(16,17)$.

PIVC insertion in the ED has been associated with higher rates of idle PIVCs ${ }^{(17-19)}$. The Cannulation Rates in the Emergency Department Intervention Trial (CREDIT) study conducted in Queensland, Australia highlighted that one-third of PIVCs inserted in the ED were idle ${ }^{(20)}$. Reducing rates of idle or never used PIVCs should be a goal of all emergency health care systems, including the pre-hospital setting.

Paramedics are an important group of providers who are skilled in PIVC insertion and are commonly the first point of healthcare contact for the patient. Sparse knowledge of PIVC insertion practices in the prehospital environment exists ${ }^{(21)}$.

The circumstances under which paramedics insert a PIVC and the number of attempts taken to complete the procedure have not been comprehensively explored within existing literature. Extrication difficulties, patient movement, bystanders and weather conditions are factors which may negatively impact successful PIVC insertion. The austere nature of pre-hospital care may lead to different decisions on when, or why, to insert a PIVC compared to clinicians in other settings such as the ED. This may have an effect on the proportion of idle PIVC compared to ED insertion. The lack of literature regarding rates of idle PIVC, especially in the pre-hospital setting has been highlighted previously ${ }^{(21)}$.

We aimed to describe contemporaneous PIVC insertion practices in pre-hospital and ED patient cohorts, with a focus on idle PIVC rates. Specifically our objectives were to: 1) describe and compare the rate of idle PIVCs between the pre-hospital and ED setting; 2) compare pre-hospital and ED cannulation practices, and 3) identify patient and clinician factors associated with idle PIVCs.

\section{Methods}

\section{Design and setting}

This was an observational cohort study in southeast Queensland, Australia, studying adult patients from both a large tertiary level emergency department (ED) and the local statutory ambulance service who had a PIVC inserted. Human research ethics committee approval, including waiver of consent, was obtained. 
The ED is a mixed adult and paediatric Level 1 trauma centre with over 110,000 ED presentations annually.

\section{Participants}

During 9 February - 18 March 2017 and 5 January - 4 February 2018, data on 1507 eligible ED presentations were prospectively collected by a research nurse ${ }^{(22)}$. Inclusion criteria were patients who were adults and either had a PIVC inserted within the ED, or by a paramedic in the pre-hospital setting. Patients who had an ATS category of $1,{ }^{(23)}$ had a PIVC inserted in another hospital and were transferred between hospitals were excluded (Fig. 1).

\section{Primary Outcome Measure}

The primary outcome measure was the idle PIVC rate, defined as the proportion of PIVCs inserted but not used within 24 hours of placement. PIVC use was defined as administration of intravenous medication, fluid or contrast. PIVC use for a "flush dose" of normal saline to ensure cannula patency and/or drawing blood from a PIVC for pathology was not defined as PIVC use. Other outcomes of interest included comparison of PIVC insertion practices, including PIVC size, anatomical insertion site and inserter staff level, as well as identifying predictors of idle PIVC.

\section{Data Sources}

For participants who were prospectively identified as having a PIVC inserted by the ambulance service, the electronic ambulance report form (eARF), ED and inpatient electronic medical records (EMRs) were retrospectively interrogated for relevant variables. From these sources, data on PIVC use within 24 hours were extracted and included: patient demographics (e.g. gender, age), PIVC characteristics (e.g. gauge, insertion site) and inserting clinician characteristics (e.g. designation, years of service) were collected (Table 1). For participants who had a PIVC inserted within the ED the same features were extracted from an existing prospectively captured data set ${ }^{(22)}$ (Table 2 ).

\section{Table 1}

Data captured from electronic ambulance report forms 


\begin{tabular}{|l|l|l|}
\hline Patient features & PIVC insertion features & Clinician features \\
\hline Demographics, age, sex & Anatomical insertion site & Years of service \\
Presenting complaint & Cannula size/gauge & Clinicians scope of \\
Triage category & Number of insertion attempts & practice* \\
Distance from scene to & Insertion success (yes/no) & Clinician medal \\
hospital (kms) & Complications $\#$ & number \\
Time from scene to hospital & PIVC use (yes/no and if yes, what was & \\
(mins) & it used for?) & \\
Time on scene (mins) & & \\
\hline
\end{tabular}

*Clinicians scope of practice refers to the paramedic's clinical level, paramedics included in the study where student paramedics, advanced care paramedics, critical care paramedics and High Acuity Response (HARU) paramedics. ${ }^{\#}$ Arterial puncture/fluid extravasation/haematoma or haemorrhage/ venous air embolus

\section{Table 2.}

Data captured from ED medical records

\begin{tabular}{|l|l|l|}
\hline PIVC insertion features & Patient features & Clinician features \\
\hline Anatomical insertion site & Demographics, age, sex & Doctor \\
Cannula size/gauge & Presenting complaint & Nurse \\
Number of insertion attempts & Triage category & \\
Insertion success (yes/no), & Time of day & \\
Complications & & \\
PIVC use (yes/no and if yes, what used for) & & \\
& & \\
\hline
\end{tabular}

ED Emergency Department; PIVC Peripheral intravenous catheter

\section{Sample Size And Data Analysis}

We calculated the sample size necessary to identify a clinically significant difference in idle PIVC rates between pre-hospital and ED settings. Based on existing literature showing the idle PIVC rate to be around $30 \%$, we considered a difference of $+/-10 \%$ to be clinically significant ${ }^{(20)}$. In order to be powered at $80 \%$ with an alpha of 0.05 , we required data on 390 patients with PIVC insertions in both pre-hospital and ED groups.

Data analysis involved both univariate and multivariate methods, using SPSS v26.0. Simple descriptive statistics (proportions, frequencies, and means of central tendency) were calculated. A 95\% confidence interval around the proportion of "idle" PIVC was calculated using statistical software (Open Epi), and the 
Wilson score method. Comparisons of proportion of idle PIVCs, and insertion practices between prehospital and ED practice employed chi-square tests. Univariate associations of potential predictors of idle PIVC were examined in the pre-hospital setting, and across the emergency setting. Crude odds ratios (OR) and $95 \%$ confidence intervals $(\mathrm{Cl})$ were calculated.

Two logistic regression models using a backwards conditional approach were developed with the dependent variable of idle PIVC. The first assessed predictors of idle PIVC for pre-hospital PIVCs only, with independent variables of age, sex, gauge, insertion site, cannulator characteristics, time (minutes) at site, time of day, distance from site to hospital, and time from scene to hospital (Table 1). The second assessed predictors of idle PIVC for all PIVCs placed in the emergency setting (pre-hospital and ED combined), with a more limited set of potential predictors that were applicable to both settings (age, sex, cannulator experience, presentation time of day, gauge, anatomical insertion site, and insertion setting). The pre-hospital specific variables, such as time on scene were not included. The ATS for the pre-hospital group was determined from the ED ATS. The number of records with missing data was presented for each variable. In all inferential univariate comparisons, the missing data category was excluded from the chi-square tests. In the multivariable modelling, records missing potential predictors were automatically excluded case-wise from the initial backwards conditional analysis. Variables that remained significant in the backwards model were then forced into a new model with only those variables. Adjusted odds ration (OR) and 95\% confidence interval (Cl) were calculated for this final model.

\section{Results}

The eligible patients and relevant exclusions are outlined in Fig. 1. Of the 1249 patients with PIVC insertions included (372 pre-hospital and 877 in ED), nearly half (45.2\%) were over 60 years of age and half $(50.1 \%)$ were female. Table 3 describes the characteristics of PIVC placed in the ED, pre-hospital a setting and combined, where Table 4 describes the pre-hospital setting only.

\section{Table 3.}

\section{Characteristics of PIVC placed in the ED, pre-hospital setting and combined.}


Characteristic of cannulation

Use within 24 hours

Used within 24 hours

Idle

883

366

Arrival mode of patient

Ambulance 674

Self-presented 575

Clinician inserting

\begin{tabular}{rc} 
Doctor & 277 \\
Nurse & 587 \\
Paramedic & 372 \\
\hline Unknown & 13
\end{tabular}

Seniority of first clinician cannulating

\begin{tabular}{|c|c|}
\hline Student & 8 \\
\hline Level 1 & 68 \\
\hline Level 2 & 997 \\
\hline Level 3 & 113 \\
\hline Unknown & 63 \\
\hline
\end{tabular}

Patient factors

Age

$$
\begin{array}{rr}
18-29 & 184 \\
\hline 30-59 & 500 \\
\hline 60+ & 565
\end{array}
$$

Sex

$$
\begin{array}{cr}
\text { F } & 626 \\
\text { M } & 623
\end{array}
$$

50.1

49.9

\section{Triage category}

$\begin{array}{rcc}1 & 0 & 0.0 \\ 2 & 503 & 40.3 \\ 3 & 661 & 52.9 \\ 4 & 74 & 5.9 \\ 5 & 3 & 0.2 \\ \text { Missing } & 8 & 0.6\end{array}$

Time category

$\begin{array}{llll}(18: 00-05: 59) & \text { Night } & & \\ & & 308 & 24.7 \\ (06: 00-17: 59) & & 941 & 75.1\end{array}$

Number of attempts at cannulation

\begin{tabular}{ccccc}
$\begin{array}{c}\text { ED } \\
\text { (n=877) }\end{array}$ & \multicolumn{2}{c}{$\begin{array}{c}\text { Pre-hospital } \\
\text { (n=372) }\end{array}$} & p-value \\
\hline $\mathbf{n}$ & $\%$ & $\mathbf{n}$ & $\%$ & \\
585 & 66.7 & 298 & 80.1 & $<0.001$ \\
292 & 33.3 & 74 & 19.9 & \\
& & & & \\
302 & 34.4 & 372 & 100.0 & N/A \\
575 & 65.6 & 0 & 0.0 & \\
& & & & \\
277 & 31.6 & NA & & \\
587 & 66.9 & NA & & N/A \\
NA & & 372 & 100.0 & \\
13 & 1.5 & 0 & 0.0 & \\
& & & & \\
\end{tabular}

$\begin{array}{ccccc} & & & & \text { Not } \\ 5 & 0.6 & 3 & 0.8 & \text { assessed } \\ 20 & 2.3 & 48 & 12.9 & \\ 741 & 84.5 & 256 & 68.8 & \\ 98 & 11.2 & 15 & 4.0 & \\ 13 & 3.5 & 50 & 13.4 & \end{array}$

$\begin{array}{ccccc}141 & 16.1 & 43 & 11.6 & 0.117 \\ 344 & 39.2 & 156 & 41.9 & \\ 392 & 44.7 & 173 & 46.5 & \end{array}$

$\begin{array}{lllll}462 & 52.7 & 164 & 44.1 & 0.005\end{array}$

$\begin{array}{llll}415 & 47.3 & 208 & 55.9\end{array}$

$\begin{array}{ccccc}0 & 0.0 & 0 & 0.0 & <0.001^{*} \\ 316 & 36.0 & 187 & 50.3 & \\ 492 & 56.1 & 169 & 45.4 & \\ 67 & 7.6 & 7 & 1.9 & \\ 2 & 0.2 & 1 & 0.3 & \\ 0 & 0.0 & 8 & 2.2 & \\ \end{array}$

$\begin{array}{lllll}201 & 22.9 & 107 & 28.8 & 0.012\end{array}$

$\begin{array}{llll}676 & 77.1 & 265 & 70.4\end{array}$

$\begin{array}{llll}684 & 78.0 & 304 & 81.7\end{array}$ 


$\begin{array}{rcc}2 & 140 & 11.2 \\ 3+ & 83 & 6.6 \\ \text { Missing } & 38 & 3.0\end{array}$

Cannula insertion site (first)

$\begin{array}{rrr}\text { Ante-cubital fossa } & 759 & 60.8 \\ \text { Dorsum } & 270 & 21.6 \\ \text { Forearm } & 116 & 9.3 \\ \text { Unknown } & 104 & 8.3\end{array}$

\begin{tabular}{|ccccc}
\hline 574 & 65.5 & 185 & 49.7 & $<0.001^{*}$ \\
175 & 20.0 & 95 & 25.5 & \\
65 & 7.4 & 51 & 13.7 & \\
63 & 7.2 & 41 & 11.0 & \\
\hline
\end{tabular}

Cannula gauge (first)

$(n=328)$

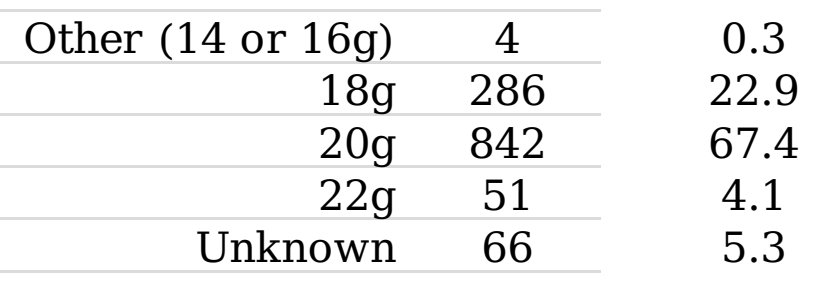

$\begin{array}{ccccc}1 & 0.1 & 3 & 0.8 & <0.001^{*} \\ 139 & 15.8 & 147 & 39.5 & \\ 675 & 77.0 & 167 & 44.9 & \\ 40 & 4.6 & 11 & 3.0 & \\ 22 & 2.5 & 44 & 11.8 & \end{array}$

\section{Purpose of PIVCs use in first 24 hours (one only)}

\begin{tabular}{|c|c|c|}
\hline Multiple non-pathology & & \\
\hline purposes & 426 & 34.1 \\
\hline Other single purpose only & 139 & 11.1 \\
\hline $\begin{array}{ll}\text { Fluids only }\end{array}$ & 130 & 10.4 \\
\hline Pain only & 83 & 6.6 \\
\hline Nausea only & 32 & 2.6 \\
\hline Used, but specific use not & 73 & 58 \\
\hline $\begin{array}{r}\text { Pathology only or no use } \\
\text { identified }\end{array}$ & 366 & 29.3 \\
\hline
\end{tabular}

Purpose of PIVC use includes (multiple options available)

\begin{tabular}{rc} 
Pain & 280 \\
Fluids & 413 \\
\hline Other IV & 550 \\
\hline Pathology & 843 \\
\hline Unsure & 73 \\
\hline
\end{tabular}

22.4

33.1

44.0

67.5

5.8

\begin{tabular}{|cccc}
320 & 36.5 & 106 & 28.5 \\
137 & 15.6 & 2 & 0.5 \\
105 & 12.0 & 25 & 6.7 \\
23 & 2.6 & 60 & 16.1 \\
$*$ & 0.0 & 32 & 8.6 \\
NA & 0.0 & 73 & 19.6 \\
292 & 33.3 & 74 & 19.9
\end{tabular}

\begin{tabular}{ccccc}
129 & 14.7 & 151 & 40.6 & $<.001^{\wedge}$ \\
365 & 41.6 & 48 & 12.9 & $<.001^{\wedge}$ \\
437 & 49.8 & 113 & 30.4 & $<.001^{\wedge}$ \\
843 & 96.1 & 0 & 0.0 & \\
0 & 0.0 & 73 & 19.6 & \\
\hline
\end{tabular}

Five attempted PIVCS not included. *Unknown/missing data excluded from inferential statistics. ^Compares ED vs pre-hospital used for this purpose vs without this purpose. Student (either setting), Level 1 (graduate paramedic, intern), Level 2: ACP II, RN, resident, Level 4: CCP, HARU, CN, CNC, consultant or registrar.

\section{Table 4.}

Characteristics of cannula placed pre-hospital, and factors associated with pre-hospital idle PIVC 


\begin{tabular}{|c|c|c|c|c|c|c|c|c|}
\hline & \multicolumn{2}{|c|}{$\begin{array}{c}\text { Total } \\
(\mathrm{n}=375)\end{array}$} & \multicolumn{2}{|c|}{$\begin{array}{l}\text { PIVC used } \\
(n=298)\end{array}$} & \multicolumn{2}{|c|}{$\begin{array}{c}\text { Idle PIVC } \\
(\mathrm{n}=74)\end{array}$} & \multirow[t]{2}{*}{ p-value } & \multirow[t]{2}{*}{$\begin{array}{l}\text { Crude OR } \\
(95 \% \text { CI })\end{array}$} \\
\hline & $\mathbf{n}$ & $\%$ & $\mathbf{n}$ & $\%$ & $\mathbf{n}$ & $\%$ & & \\
\hline \multicolumn{9}{|l|}{ Age } \\
\hline $18-29$ & 43 & 11.5 & 34 & 79.1 & 9 & 20.9 & 0.867 & $1.2(0.7-2.0)$ \\
\hline $30-59$ & 158 & 42.1 & 127 & 81.4 & 29 & 18.6 & & $1.0(0.4-2.3)$ \\
\hline $60+$ & 174 & 46.4 & 137 & 79.2 & 36 & 20.8 & & 1.0 (reference) \\
\hline \multicolumn{9}{|l|}{ Sex } \\
\hline $\mathrm{F}$ & 165 & 44.0 & 131 & 79.9 & 33 & 20.1 & 0.922 & $1.0(0.6-1.7)$ \\
\hline $\mathrm{M}$ & 210 & 56.0 & 167 & 80.3 & 41 & 19.7 & & 1.0 (reference) \\
\hline \multicolumn{9}{|l|}{ Triage categorył } \\
\hline & 0 & 0.0 & 0 & NA & 0 & NA & 0.122 & Not calculated \\
\hline & 188 & 50.1 & 150 & 79.8 & 37 & 19.8 & & \\
\hline 3 & 171 & 45.6 & 134 & 45.0 & 35 & 20.7 & & \\
\hline 4 & 7 & 1.9 & 7 & 100.0 & 0 & 0.0 & & \\
\hline 5 & 1 & 0.3 & 0 & 0.0 & 1 & 100.0 & & \\
\hline Missing & 8 & 2.1 & 7 & 87.5 & 1 & 12.5 & & \\
\hline \multicolumn{9}{|l|}{$\begin{array}{l}\text { Presenting } \\
\text { complaint }\end{array}$} \\
\hline Chest Pain & 109 & 29.1 & 82 & 76.6 & 25 & 23.4 & $\begin{array}{l}\text { Not } \\
\text { calculated }\end{array}$ & Not calculated \\
\hline Pain & 75 & 20.0 & 69 & 93.2 & 5 & 6.8 & & \\
\hline Neurological & 54 & 14.4 & 41 & 75.9 & 13 & 24.1 & & \\
\hline Trauma & 40 & 10.7 & 31 & 77.5 & 9 & 22.5 & & \\
\hline Other & 34 & 9.1 & 21 & 61.8 & 13 & 38.2 & & \\
\hline Dyspnoea & 27 & 7.2 & 25 & 92.6 & 2 & 7.4 & & \\
\hline Gastro-intestinal & 13 & 3.5 & 13 & 100.0 & 0 & 0.0 & & \\
\hline Seizure & 13 & 3.5 & 9 & 69.2 & 4 & 30.8 & & \\
\hline Diabetic Related & 5 & 1.3 & 4 & 80.0 & 1 & 20.0 & & \\
\hline Sepsis & 5 & 1.3 & 3 & 60.0 & 2 & 40.0 & & \\
\hline \multicolumn{9}{|l|}{ Complaint } \\
\hline $\begin{array}{r}\text { Pain or chest } \\
\text { pain }\end{array}$ & 184 & 49.1 & 151 & 83.4 & 30 & 16.6 & 0.119 & 1.0 (reference) \\
\hline Any other & 191 & 50.9 & 147 & 77.0 & 44 & 23.0 & 0.115 & $1.5(0.9-2.5)$ \\
\hline \multicolumn{9}{|l|}{ Time categorył } \\
\hline $\begin{array}{r}\text { Night (18:00- } \\
05: 59)\end{array}$ & 108 & 28.8 & 89 & 83.2 & 18 & 16.8 & 0.346 & 1.0 (reference) \\
\hline $\begin{array}{r}\text { Day }(06: 00- \\
17: 59)\end{array}$ & 264 & 70.4 & 206 & 78.6 & 56 & 21.4 & & $1.3(0.7-2.4)$ \\
\hline \multicolumn{9}{|l|}{$\begin{array}{l}\text { Time on scene } \neq \\
\text { (minutes) }\end{array}$} \\
\hline$<15$ minutes & 35 & 9.3 & 27 & 79.4 & 7 & 20.6 & 0.663 & $1.2(0.5-3.2)$ \\
\hline 15-29 minutes & 214 & 57.1 & 167 & 78.0 & 47 & 22.0 & & $1.3(0.7-2.3)$ \\
\hline $30+$ & 115 & 30.7 & 93 & 82.3 & 20 & 17.7 & & 1.0 (reference) \\
\hline Missing & 11 & 3.2 & 11 & 91.7 & 0 & 0.0 & & \\
\hline \multicolumn{9}{|c|}{$\begin{array}{l}\text { Time in transit } ₹ \text { (depart site } \\
\text { to ED triaqe) }\end{array}$} \\
\hline$<15$ minutes & 116 & 30.9 & 95 & 81.9 & 21 & 18.1 & 0.257 & 1.0 (reference) \\
\hline
\end{tabular}


$\begin{array}{rcc}15-29 \text { minutes } & 197 & 52.5 \\ 30+ & 53 & 14.1 \\ \text { Missing } & 9 & 2.4\end{array}$

Total time pre-

hospitalł

$(n=366)$

\begin{tabular}{rcc}
$<30$ & 31 & 8.3 \\
$30-44$ & 154 & 41.1 \\
$45-59$ & 129 & 34.4 \\
$60+$ & 55 & 14.7 \\
\cline { 1 - 2 } Missing & 6 & 1.6
\end{tabular}

Distance to hospital

(kms) ₹

\begin{tabular}{rcc|}
$r<10$ & 184 & 49.1 \\
$10-19$ & 124 & 33.1 \\
\cline { 1 - 2 } $20+$ & 63 & 16.8 \\
\cline { 1 - 2 } Missing & 4 & 1.1
\end{tabular}

Number of attemptsł

$(n=334)$

$\begin{array}{rcc}1 & 307 & 81.9 \\ 2 & 22 & 5.9 \\ 3+ & 8 & 2.1 \\ \text { Missing } & 38 & 10.1\end{array}$

\section{Insertion site}

(first) $¥$

$\begin{array}{rrr}\text { Ante-cubital } & & \\ \text { fossa } & 185 & 49.3 \\ \text { Dorsum } & 95 & 25.3 \\ \text { Forearm } & 51 & 13.6 \\ \text { Missing } & 44 & 11.7\end{array}$

Gauge (first) $\ddagger^{\wedge}$

$\begin{array}{rcc}16 g & 3 & 0.8 \\ 18 g & 147 & 39.2 \\ 20 g & 167 & 44.5 \\ 22 \mathrm{~g} & 11 & 2.9 \\ \text { Missing } & 47 & 12.5\end{array}$

\section{Skillset‡ ${ }^{\#}$}

\section{(first cannulator)}

$\begin{array}{rcc}\text { ACP II } & 256 & 68.3 \\ \text { CCP } & 14 & 3.7 \\ \text { Graduate } & & \\ \text { Paramedic } & 48 & 12.8 \\ \text { HARU } & 1 & 0.3 \\ \text { Student } & 3 & 0.8 \\ \text { Missing } & 53 & 14.1\end{array}$

Years of service (first cannulator)

$$
\begin{array}{lll}
<2 \text { years } & 67 & 17.9
\end{array}
$$

$\begin{array}{cccc}157 & 80.5 & 38 & 19.5 \\ 37 & 71.2 & 15 & 28.8 \\ 9 & 100.0 & 0 & 0.0\end{array}$

$1.1(0.6-2.0)$

$1.8(0.9-3.9)$

\begin{tabular}{||cccccl}
\hline 24 & 77.4 & 7 & 22.6 & 0.152 & $0.9(0.3-2.7)$ \\
117 & 76.0 & 37 & 24.0 & & $1.0(0.5-2.1)$ \\
109 & 86.5 & 17 & 13.5 & & $0.5(0.2-1.1)$ \\
42 & 76.4 & 13 & 23.6 & & 1.0 (reference) \\
6 & 100.0 & 0 & 0.0 & & \\
& & & & & \\
& & & & & \\
146 & 80.2 & 36 & 19.8 & 0.978 & 1.0 (reference) \\
98 & 79.7 & 25 & 20.3 & & $1.0(0.6-1.8)$ \\
51 & 82.3 & 12 & 19.0 & & $1.0(0.5-2.0)$ \\
3 & 75.0 & 1 & 25.0 & & NA \\
& & & & & \\
& & & & & \\
249 & 81.9 & 55 & 18.1 & 0.806 & Not calculated \\
19 & 86.4 & 3 & 13.6 & & \\
7 & 87.5 & 1 & 12.5 & & NA \\
23 & 60.5 & 15 & 39.5 & &
\end{tabular}

\begin{tabular}{cccccl}
155 & 83.8 & 30 & 16.2 & 0.689 & 1.0 (reference) \\
\hline 76 & 80.0 & 19 & 20.0 & & $1.3(0.7-2.4)$ \\
41 & 80.4 & 10 & 19.6 & & $1.6(0.6-2.8)$ \\
26 & 59.1 & 15 & 34.1 & &
\end{tabular}

$\begin{array}{cccccl}1 & 33.3 & 2 & 66.7 & 0.141 & 1.0 \text { (reference) } \\ 124 & 84.4 & 23 & 15.6 & & 1.1(0.6-2.0) \\ 136 & 81.4 & 31 & 18.6 & & \\ 9 & 81.8 & 2 & 18.2 & & \\ 28 & 59.6 & 19 & 40.4 & & \end{array}$

$\begin{array}{llllll}214 & 83.6 & 42 & 16.4 & 0.551 & \text { Not calculated }\end{array}$ $\begin{array}{llll}11 & 78.6 & 3 & 21.4\end{array}$

$\begin{array}{llll}36 & 75.0 & 12 & 25.0\end{array}$

$\begin{array}{llll}1 & 100.0 & 0 & 0.0\end{array}$

$\begin{array}{llll}3 & 100.0 & 0 & 0.0\end{array}$

$\begin{array}{llll}33 & 62.3 & 17 & 32.1\end{array}$
0.15

$0.9(0.4-1.9)$ 


\begin{tabular}{rcc||ccccl}
$2-4$ years & 57 & 15.2 & 52 & 91.2 & 5 & 8.8 & $0.3(0.1-0.9)$ \\
5-9 years & 94 & 25.1 & 79 & 84.0 & 15 & 16.0 & $0.6(0.3-1.3)$ \\
$10+$ years & 100 & 26.7 & 77 & 77.0 & 23 & 23.0 & 1.0 (reference) \\
Missing & 57 & 15.2 & 37 & 64.9 & 17 & 29.8 & \\
\hline
\end{tabular}

Three unsuccessful cannulations are included in the totals column only. *unknown/missing category not included in inferential statistics. ^ comparing 20-22 vs $\leq 18 \mathrm{~g} .{ }^{\#}$ ACP II= Advanced Care Paramedic level 2, CCP $=$ Critical Care Paramedic, Graduate Paramedic $=$ paramedic during their internship HARU= High Acuity Response Paramedic, Student= student paramedic on university placement.

\section{Idle PIVC rates}

The overall idle PIVC rate in the emergency setting (pre-hospital and ED combined) was $29.3 \%(n=366)$, 95\% Cl: 26.9\%-31.9\% (Table 3). Of the 372 patients who had their PIVC inserted prehospitally, 1 in 5 $(19.9 \%, n=74)$ had an idle PIVC, whereas one-third $(33.3 \%, n=292)$ of the patients who had a PIVC in ED had an idle PIVC. Nearly $40 \%(39.5 \%, n=147)$ of PIVCs inserted in the pre-hospital setting were not used by paramedics whilst in the ambulance. Nursing staff were most likely to insert a PIVC that remained idle $(35.1 \%, n=206)$ compared to doctors $(29.6 \%, n=82)$ and paramedics $(19.9 \%, n=74)$ (Table 5).

\section{Comparison between pre-hospital and ED}

Table 3 outlines the comparison between pre-hospital and ED for PIVC insertion. Women represented a smaller proportion of PIVCs placed by pre-hospital clinicians (44.1\% vs $55.9 \%$ ). In addition, PIVCs placed pre-hospital were more likely to be in more urgent ATS categories, with $50.3 \%$ from ATS 2 . While PIVCs placed within the ED included fewer patients with ATS 2 (36.0\%) and more ATS 3, 4 and 5 compared to pre-hospital PIVCs. For PIVCs inserted in the ED $96.1 \%$ were used for pathology, with $33.3 \%$ used only for pathology (or no use identified). Although both ED and pre-hospital clinicians inserted most PIVCs in the antecubital fossa, pre-hospital staff were more likely to use the forearm $(13.7 \%$ vs $7.4 \%$, $(p<.001)$. Prehospital staff documented fewer attempts at cannulation (range 1-4, 9.0\% with two or more attempts), compared to ED (range $1-10,22 \%$ with two or more attempts), $(p<.001)$. No PIVC complications were recorded on ambulance eARFs.

\section{Pre-hospital PIVC}

The most common reasons PIVCs were placed pre-hospital were chest pain and other types of pain (Table 4). Of those 372 PIVCs successfully placed pre-hospital, 225 (60.5\%) were used by the ambulance service, mostly for administration of pain relief $(n=151)$. Approximately half $(n=112,49.8 \%)$ of all patients who had their PIVCs used pre-hospital had intravenous anti-emetics administered through them.

\section{Predictors of idle PIVC}

Within the pre-hospital cohort a logistic regression multivariable model did not find any significant predictors of idle PIVCs amongst the characteristics studied, including distance or time to hospital (Table 4). Factors univariately associated with increased idle PIVC rate for the emergency setting (pre- 
hospital and ED combined) are outlined in Table 5 and included; PIVCs insertion in ED, by doctors or nurses, patients who self-presented, insertion during the day (0600-1759 hrs) and insertion in places other than the forearm. In the multivariable logistic regression analysis, ED PIVC insertion (OR 2.4, 95\% Cl 1.7-3.3) and triage category (ATS 2 and 3 vs 4 and 5) (OR 0.7, 95\% Cl 0.5-0.8), were the only factors significantly predicting idle PIVC (Table 5).

\section{Table 5.}

Characteristics of PIVC inserted (ED and pre-hospital combined), and factors associated with idle PIVC 


\begin{tabular}{|c|c|c|c|c|c|c|c|c|c|}
\hline $\begin{array}{l}\text { Characteristics } \\
\text { of PIVC }\end{array}$ & \multicolumn{2}{|c|}{$\begin{array}{c}\text { All } \\
\text { emergency } \\
\text { PIVCs } \\
(\mathrm{n}=1249)\end{array}$} & \multicolumn{2}{|c|}{$\begin{array}{c}\text { Used } \\
\text { within 24 } \\
\text { hours } \\
\text { (n=883) }\end{array}$} & \multicolumn{2}{|c|}{$\begin{array}{c}\text { Idle } \\
(\mathrm{n}=366)\end{array}$} & p-value & $\begin{array}{l}\text { Crude OR } \\
(95 \% \text { CI })\end{array}$ & $\begin{array}{c}\begin{array}{c}\text { Adjusted OR } \\
(95 \% \mathrm{CI})\end{array} \\
\end{array}$ \\
\hline $\begin{array}{l}\text { Geographical } \\
\text { location of } \\
\text { insertion }\end{array}$ & $\mathrm{n}$ & $\begin{array}{l}\text { valid } \\
\%\end{array}$ & $\mathrm{n}$ & $\%$ & $\mathrm{n}$ & $\%$ & & & \\
\hline Pre-hospital & 372 & 29.8 & 298 & 80.1 & 74 & 19.9 & $<0.001$ & $\begin{array}{c}1.0 \\
\text { (reference) }\end{array}$ & $\begin{array}{l}1.0 \\
\text { (reference) }\end{array}$ \\
\hline ED & 877 & 70.2 & 585 & 66.7 & 292 & 33.3 & & & $\begin{array}{l}2.4(1.7- \\
3.3)\end{array}$ \\
\hline \multicolumn{10}{|l|}{ Staff type } \\
\hline Nurse & 587 & 47.0 & 381 & 64.9 & 206 & 35.1 & $<0.001$ & $\begin{array}{l}2.5(1.8- \\
3.5)\end{array}$ & $\begin{array}{l}\text { Not tested } \\
\text { in model }\end{array}$ \\
\hline Doctor & 277 & 22.2 & 195 & 70.4 & 82 & 29.6 & & $\begin{array}{l}2.0(1.3- \\
2.9)\end{array}$ & \\
\hline Paramedic & 372 & 29.8 & 298 & 80.1 & 74 & 19.9 & & $\begin{array}{l}1.0 \\
\text { (reference) }\end{array}$ & \\
\hline Other ED staff & 13 & 1.0 & 9 & 69.2 & 4 & 30.8 & & & \\
\hline \multicolumn{10}{|l|}{$\begin{array}{l}\text { Arrival mode of } \\
\text { patient }\end{array}$} \\
\hline Pre-hospital & 674 & 54.0 & 504 & 74.8 & 170 & 25.2 & 0.001 & (reference) & $\begin{array}{l}\text { Not tested } \\
\text { in model }\end{array}$ \\
\hline Self-present & 575 & 46.0 & 379 & 65.9 & 196 & 34.1 & & $2.0)$ & \\
\hline \multicolumn{10}{|l|}{$\begin{array}{l}\text { Seniority (first } \\
\text { clinician) }\end{array}$} \\
\hline Student & 8 & 0.6 & 7 & 87.5 & 1 & 12.5 & & calculated & $\begin{array}{l}\text { Not tested } \\
\text { in model }\end{array}$ \\
\hline Level 1 & 68 & 5.4 & 50 & 73.5 & 18 & 26.5 & & & \\
\hline Level 2 & 997 & 79.8 & 702 & 70.4 & 295 & 29.6 & & & \\
\hline Level 3 & 113 & 9.0 & 82 & 72.6 & 31 & 27.4 & & & \\
\hline Unknown & 63 & 5.0 & 42 & 66.7 & 21 & 33.3 & & & \\
\hline \multicolumn{10}{|l|}{ Age } \\
\hline $18-29$ & 184 & 14.7 & 120 & 65.2 & 64 & 34.8 & 0.05 & $\begin{array}{l}1.4(1.0- \\
2.1)\end{array}$ & $\begin{array}{l}\text { Not } \\
\text { retained in } \\
\text { model }\end{array}$ \\
\hline $30-59$ & 500 & 40.0 & 351 & 70.2 & 149 & 29.8 & 0.33 & $1.5)^{1.9-}$ & \\
\hline $60+$ & 565 & 45.2 & 412 & 72.9 & 153 & 27.1 & 0.13 & $\begin{array}{l}\text { l.0 } \\
\text { (reference) }\end{array}$ & \\
\hline $\mathrm{F}$ & 626 & 50.1 & 438 & 70.0 & 188 & 30.0 & 0.571 & $\begin{array}{l}1.1(0.8- \\
1.4)\end{array}$ & $\begin{array}{l}\text { Not } \\
\text { retained in } \\
\text { model }\end{array}$ \\
\hline $\mathrm{M}$ & 623 & 49.9 & 445 & 71.4 & 178 & 28.6 & & (reference) & \\
\hline $\begin{array}{l}\text { Triage category } \\
\ddagger €\end{array}$ & & & & & & & 0.04 & $\begin{array}{l}0.8(0.7- \\
1.0)\end{array}$ & $\begin{array}{l}0.7(0.5- \\
0.8)\end{array}$ \\
\hline 1 & 0 & 0.0 & 0 & NA & 0 & 0.0 & & & \\
\hline 2 & 503 & 40.3 & 340 & 67.6 & 163 & 32.4 & & & \\
\hline 3 & 661 & 52.9 & 477 & 72.2 & 184 & 27.8 & & & \\
\hline
\end{tabular}




\begin{tabular}{|c|c|c|c|c|c|c|c|c|c|}
\hline 4 & 74 & 5.9 & 57 & 77.0 & 17 & 23.0 & & & \\
\hline 5 & 3 & 0.2 & 2 & 66.7 & 1 & 33.3 & & & \\
\hline Missing & 8 & 0.6 & 7 & 87.5 & 1 & 12.5 & & & \\
\hline \multicolumn{10}{|l|}{ Time category } \\
\hline $\begin{array}{c}\text { Night (18:00- } \\
05: 59)\end{array}$ & 308 & 24.7 & 232 & 75.3 & 76 & 24.7 & 0.04 & \multirow{2}{*}{$\begin{array}{l}1.0 \\
\text { (reference) } \\
1.4(1.0- \\
1.8)\end{array}$} & \multirow[t]{2}{*}{$\begin{array}{l}\text { Not } \\
\text { retained in } \\
\text { model }\end{array}$} \\
\hline $\begin{array}{l}\text { Day } \\
\text { (06:00-17:59) }\end{array}$ & 941 & 75.3 & 651 & 69.2 & 290 & 30.9 & & & \\
\hline \multicolumn{2}{|c|}{ Number of attempts $¥$} & & & & & & & \multirow{5}{*}{$\begin{array}{l}\text { Not } \\
\text { calculated }\end{array}$} & \multirow{5}{*}{$\begin{array}{l}\text { Not tested } \\
\text { in model }\end{array}$} \\
\hline 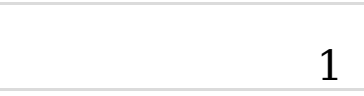 & 988 & 79.1 & 690 & 69.8 & 298 & 30.2 & 0.163 & & \\
\hline 2 & 140 & 11.2 & 107 & 76.4 & 33 & 23.6 & & & \\
\hline $3+$ & 83 & 6.6 & 63 & 75.9 & 20 & 24.1 & & & \\
\hline Missing & 38 & 3.0 & 23 & 60.5 & 15 & 39.5 & & & \\
\hline \multicolumn{10}{|l|}{$\begin{array}{l}\text { Cannula } \\
\text { insertion site‡ } \\
\text { (first) }\end{array}$} \\
\hline $\begin{array}{r}\text { Ante-cubital } \\
\text { fossa }\end{array}$ & 758 & 60.8 & 525 & 69.3 & 233 & 30.7 & 0.049 & \multirow{4}{*}{$\begin{array}{l}1.5(0.9- \\
2.6) \\
1.8(1.1- \\
2.9) \\
1.0 \\
\text { (reference) }\end{array}$} & \multirow{4}{*}{$\begin{array}{l}1.6(1.0- \\
2.6) \\
1.1(0.8- \\
1.5) \\
1.0 \\
\text { (reference) }\end{array}$} \\
\hline Dorsum & 269 & 21.6 & 195 & 72.5 & 74 & 27.5 & & & \\
\hline Forearm & 116 & 9.3 & 93 & 80.2 & 23 & 19.8 & & & \\
\hline Unknown/other & 106 & 8.3 & 70 & 66.0 & 36 & 34.0 & & & \\
\hline \multicolumn{2}{|c|}{$\begin{array}{l}\text { Cannula gauge (first) } \\
\ddagger^{\wedge}(n=328)\end{array}$} & & & & & & 0.039 & \multirow{5}{*}{$\begin{array}{l}1.0 \\
\text { (reference) }\end{array}$} & \multirow{3}{*}{$\begin{array}{l}\text { Not } \\
\text { retained in } \\
\text { model }\end{array}$} \\
\hline $\begin{array}{r}\text { Other }\left(\begin{array}{r}14 \text { or } \\
16 \mathrm{~g})\end{array}\right.\end{array}$ & 4 & 0.3 & 2 & 50.0 & 2 & 50.0 & & & \\
\hline $18 \mathrm{~g}$ & 286 & 22.9 & 218 & 76.2 & 68 & 23.8 & & & \\
\hline $20 \mathrm{~g}$ & 842 & 67.4 & 584 & 69.4 & 258 & 30.6 & & & \\
\hline $22 \mathrm{~g}$ & 51 & 4.1 & 37 & 72.5 & 14 & 27.5 & & & \\
\hline Unknown & 66 & 5.3 & 42 & 63.6 & 24 & 36.4 & & & \\
\hline
\end{tabular}

Five attempted PIVCS not included. $¥$ Unknown/missing category not included in inferential statistics. €Triage category treated as an ordinal variable, classified as 2, 3 and 4 or 5 . ^ comparing 20-22 vs $\leq 18 \mathrm{~g}$. Variables marked as not retained in model dropped out of the multivariable model after adjustment for predictors with greater estimates of effect. Variables marked as not tested were not tested in the model as they were not comparable across the ED and pre-hospital setting, did not make clinical sense as a predictor, or were highly correlated with variables already in the model. Student (either setting), Level 1 (graduate paramedic, intern), Level 2: ACP II, RN, resident, Level 4: CCP, HARU, CN, CNC, consultant or registrar.

\section{Discussion}


Across the emergency setting, including pre-hospital and ED, nearly 3 in 10 (29.3\%) PIVCs placed remained idle. ED clinicians were 2.4 times more likely than paramedics to insert a PIVC that was not used within 24 hours of insertion. These findings suggest that paramedics in our study made sound decisions on when to insert a PIVC that was likely to be used. Although this left $19.9 \%$ of pre-hospital PIVCs that were not used within the first 24 hours, our results for pre-hospital PIVC insertions align with recent literature that advocates for clinicians not to insert a PIVC unless they are over $80 \%$ certain it will be used ${ }^{(20)}$.

We also demonstrated that a substantial proportion (39.5\%) of PIVCs inserted by paramedics were unused while in the pre-hospital setting. Comparatively, we report a lower percentage of unused prehospital PIVCs than previous studies which report rates as high as $72-83 \%(24,25)$. The rate of unused prehospital PIVCs in this study is of importance and prompts questions surrounding paramedics' motives for inserting pre-hospital PIVCs. Further investigation is warranted to achieve a comprehensive understanding of pre-hospital decision making regarding PIVC insertion.

The provision of symptomatic treatment in the pre-hospital environment is an essential aspect of patient care. Although analgesia provision is common and evidence-based ${ }^{(26)}$, the role of antiemetics for treating nausea via the intravenous route is not as well defined ${ }^{(27)}$. We found that pre-hospital PIVCs were most frequently used for analgesia and treating nausea. Approximately half $(49.8 \%)$ of all PIVCs inserted and used pre-hospital had intravenous anti-emetics (mainly ondansetron) administered through them; we therefore hypothesise that a number of these PIVCs were inserted solely for the purpose of administering anti-emetics. Since October 2017, paramedics in our setting have obtained access to ondansetron oral disintegrating tablets in addition to the long-standing availability of intramuscular anti-emetics. It is possible that a subset of PIVC insertions for administering anti-emetics were clinically not indicated, and even unnecessary.

The number of PIVC insertion attempts differed between the pre-hospital and ED healthcare providers. ED practitioners recorded more and a wider range of attempts than paramedics with ranges of 1-10 and 14 attempts respectively. Clinical guidelines for paramedics in our study recommend that a maximum of three PIVC insertion attempts are permitted per officer. It is likely that these guidelines, transport factors and time constraints consistent with the pre-hospital environment contributed to lower numbers of insertion attempts by paramedics. Conversely, many more attempts were taken for patients within ED requiring intravenous access; this may be due to an emergent requirement for further diagnostic imaging or pharmacology.

PIVCs inserted within ED were frequently used for obtaining blood samples (96.1\%) and in one-third of the patients the PIVC was used for blood sampling only. This study excluded pathology collection as an appropriate PIVC use due to the procedure carrying increased risks to the patient and a higher rate of complications when compared with venepuncture ${ }^{(28)}$. It is likely that patients, especially with less urgent ATS categories within our sample had a PIVC inserted in ED solely for the purpose of pathology collection. The large proportion of PIVCs being used for pathology collection in our study is congruent 
with other studies ${ }^{(16,29)}$. Egerton-Warburton et al reported a multimodal approach aimed at reducing inappropriate PIVC insertions ${ }^{(29)}$. This approach was successful in reducing unnecessary PIVCs, but not in lowering rates of PIVCs inserted for pathology collection only ${ }^{(29)}$. The financial burden and increased patient risks of PIVC placement as opposed to venepuncture must be carefully considered prior to deciding which procedure is to be performed.

Our study defined an idle PIVC as unused within 24 hours of insertion, excluding a normal saline flush or the collection of pathology via the PIVC. Previous studies have adopted various definitions of an idle PIVC; however, the most frequently used definition is consistent with ours, namely a PIVC that was inserted and remained unused ${ }^{(30)}$. It can be argued that clinically indicated PIVCs may become idle due to rapid changes in the patient's clinical condition or haemodynamic status. The complexities of patient physiology may create uncertainty around the definition of an appropriate PIVC and a zero incidence of idleness is not realistic. This imprecision has resulted in the absence of an accepted definition of a clinically indicated, yet unused PIVC. As such we deemed any idle PIVC in our study as an inappropriate insertion. A recent systematic review highlighted the lack of literature surrounding the rate of idle PIVCs in the prehospital setting ${ }^{(21)}$. Our study is the first study to directly compare idle rates of PIVC between prehospital and ED settings. This work demonstrates current practices of PIVC insertion across the broader emergency setting and lays foundations for future work about the clinical decision making of clinicians around PIVC insertion.

\section{Limitations}

This was a single setting observational study and as a result, data may not be generalisable with other populations. While the ED PIVC data was prospectively collected, pre-hospital predictor variables were retrospectively collected. Documentation of PIVC attempts is mandatory in both the pre-hospital and ED environments; however, inaccuracy in these records may be present, such as the number of recorded attempts before successful insertion. The lack of complications recorded by pre-hospital PIVCs may also reflect this. We did not collect any data on the reasoning of the PIVC inserting clinician, limiting our ability to comment on the proportion of idle PIVC which may have been clinically indicated and appropriately inserted.

\section{Conclusion}

The rate of idle PIVC within the broader emergency health care setting in this study was $29 \%$. This study provides insight into pre-hospital and ED PIVC insertion practices and highlights the differences in rates of idle PIVC between both cohorts, with PIVCs inserted in the ED 2.4 times more likely to remain idle compared to those inserted pre-hospital. Reasons for the differences in these practices are not well understood and requires more targeted research. We recommend a qualitative approach with clinicians of different backgrounds and experience to capture a more in-depth insight into clinician perceptions regarding PIVC insertion and use, guided by the findings of this study. Reducing the rate of idle PIVC 
insertion will lead to less risk and discomfort for patients and cost savings for healthcare systems, and as such this should be the goal of all healthcare providers.

\section{List Of Abbreviations}

PIVC - Peripheral Intravenous Cannula

ED- Emergency Department

ATS - Australasian Triage Scale

BSI - Blood Stream Infections

CREDIT - Canulation Rates in the Emergency Department Intervention Trial

eARF - Electronic Ambulance Report Form

EMR - Electronic Medical Records

\section{Declarations}

\section{Ethics approval and consent to participate}

Human research ethics committee approval, including waiver of consent, was obtained from Gold Coast University Hospital, and Griffith University. Queensland Ambulance Services, information support, research \& evaluation department also endorsed this project. HREC/2019/QGC/53353.

\section{Consent for publication}

Not applicable

\section{Availability of data and materials}

The datasets generated and/or analysed during the current study are not publicly available due to local ethics and governance regulations but are available from the corresponding author on reasonable request.

\section{Competing interests}

NM reports investigator-initiated research grants and speaker fees provided to Griffith University from vascular access product manufacturers (Becton Dickinson, 3M and Cardinal Health); and a consultancy payment for expert advice from Becton Dickinson.

All other authors declare that they have no competing interests. 


\section{Funding}

This study received funding from the Emergency Medicine Foundation (EMF).

\section{Authors' contributions}

$\mathrm{HE}$, developed concept, led project and wrote manuscript. AS performed data analysis, assisted with concept development, developed tables and assisted with manuscript writing. JR assisted with concept development and with manuscript writing. MC performed data collection and assisted with manuscript writing. NM provided intellectual input, content expertise and assisted with manuscript preparation. CR provided intellectual input, content expertise, interpretation of results and assisted with manuscript preparation. JB provided intellectual input and assisted with manuscript preparation. PC provided intellectual input, content expertise, and with manuscript preparation. GK assisted with concept development, manuscript writing and supervised HE.

\section{Acknowledgements}

We would like thank Dr Stuart Watkins and Dr Amy Archer Jones for providing valuable input regarding PIVC practices, as well as Dr Stephen Rashford and Dr Emma Bosley for their support.

\section{Authors information}

$\mathrm{N} / \mathrm{A}$

\section{References}

1. Bahl A, Hang B, Brackney A, Joseph S, Karabon P, Mohammad A, et al. Standard long IV catheters versus extended dwell catheters: A randomized comparison of ultrasound-guided catheter survival. Am J Emerg Med. 2019;37(4):715-21.

2. Dychter SS, Gold DA, Carson D, Haller M. Intravenous therapy: a review of complications and economic considerations of peripheral access. J Infus Nurs. 2012;35(2):84-91.

3. Zingg W, Pittet D. Peripheral venous catheters: an under-evaluated problem. Int J Antimicrob Agents. 2009;34(Suppl 4):38-42.

4. Cooke M, Ullman AJ, Ray-Barruel G, Wallis M, Corley A, Rickard CM. Not "just" an intravenous line: Consumer perspectives on peripheral intravenous cannulation (PIVC). An international crosssectional survey of 25 countries. PLoS One. 2018;13(2):e0193436.

5. Sabri A, Szalas J, Holmes KS, Labib L, Mussivand T. Failed attempts and improvement strategies in peripheral intravenous catheterization. Biomed Mater Eng. 2013;23(1-2):93-108.

6. Marsh N, Webster J, Larson E, Cooke M, Mihala G, Rickard CM. Observational Study of Peripheral Intravenous Catheter Outcomes in Adult Hospitalized Patients: A Multivariable Analysis of Peripheral Intravenous Catheter Failure. J Hosp Med. 2018;13(2):83-9. 
7. Abolfotouh MA, Salam M, Bani-Mustafa A, White D, Balkhy HH. Prospective study of incidence and predictors of peripheral intravenous catheter-induced complications. Ther Clin Risk Manag. 2014;10:993-1001.

8. Yagnik L, Graves A, Thong K. Plastic in patient study: Prospective audit of adherence to peripheral intravenous cannula monitoring and documentation guidelines, with the aim of reducing future rates of intravenous cannula-related complications. Am J Infect Control. 2017;45(1):34-8.

9. Goransson KE, Johansson E. Prehospital peripheral venous catheters: a prospective study of patient complications. J Vasc Access. 2012;13(1):16-21.

10. Rickard CM, Webster J, Wallis MC, Marsh N, McGrail MR, French V, et al. Routine versus clinically indicated replacement of peripheral intravenous catheters: a randomised controlled equivalence trial. Lancet. 2012;380(9847):1066-74.

11. Stuart RL, Cameron DR, Scott C, Kotsanas D, Grayson ML, Korman TM, et al. Peripheral intravenous catheter-associated Staphylococcus aureus bacteraemia: more than 5 years of prospective data from two tertiary health services. Med J Aust. 2013;198(10):551-3.

12. Maki DG, Kluger DM, Crnich CJ. The risk of bloodstream infection in adults with different intravascular devices: a systematic review of 200 published prospective studies. Mayo Clin Proc. 2006;81(9):1159-71.

13. Decker K, Ireland S, O'Sullivan L, Boucher S, Kite L, Rhodes D, et al. Peripheral intravenous catheter insertion in the Emergency Department. Australas Emerg Nurs J. 2016;19(3):138-42.

14. O'Grady NP, Alexander M, Burns LA, Dellinger EP, Garland J, Heard SO, et al. Guidelines for the prevention of intravascular catheter-related infections. Am J Infect Control. 2011;39(4 Suppl 1):1-34.

15. Mermel LA. Short-term Peripheral Venous Catheter-Related Bloodstream Infections: A Systematic Review. Clin Infect Dis. 2017;65(10):1757-62.

16. Limm El, Fang X, Dendle C, Stuart RL, Egerton Warburton D. Half of all peripheral intravenous lines in an Australian tertiary emergency department are unused: pain with no gain? Ann Emerg Med. 2013;62(5):521-5.

17. Gledstone-Brown L, McHugh D. Review article: Idle 'just-in-case' peripheral intravenous cannulas in the emergency department: Is something wrong? Emerg Med Australas. 2018;30(3):309-26.

18. Carr PJ, Rippey JCR, Cooke ML, Higgins NS, Trevenen ML, Foale A, et al. Derivation of a clinical decision-making aid to improve the insertion of clinically indicated peripheral intravenous catheters and promote vessel health preservation. An observational study. PLoS One. 2019;14(3):e0213923.

19. Carr PJ, Rippey JCR, Cooke ML, Higgins NS, Trevenen M, Foale A, et al. From insertion to removal: A multicenter survival analysis of an admitted cohort with peripheral intravenous catheters inserted in the emergency department. Infect Control Hosp Epidemiol. 2018;39(10):1216-21.

20. Hawkins T, Greenslade JH, Suna J, Williams J, Rickard CM, Jensen M, et al. Peripheral Intravenous Cannula Insertion and Use in the Emergency Department: An Intervention Study. Acad Emerg Med. 2018;25(1):26-32. 
21. Mason MF, Wallis M, Lord B, Barr N. Prehospital use of peripheral intravenous catheters and intraosseous devices: An integrative literature review of current practices and issues. Australasian Emergency Care. 2020;23(3):7.

22. Archer-Jones A, Sweeny A, Schults JA, Rickard CM, Johnson L, Gunter A, et al. Evaluating an ultrasound-guided peripheral intravenous cannulation training program for emergency clinicians: An Australian perspective. Australas Emerg Care. 2020;23(3):151-6.

23. Guidelines on the implementation of the Australasian Triage Scale In emergency departments [Internet]. Australasian College For Emergency Medicine. 2000 [cited 12/07/2020]. Available from: https://acem.org.au/getmedia/51dc74f7-9ff0-42ce-872a0437f3db640a/G24_04_Guidelines_on_Implementation_of_ATS_Jul-16.aspx.

24. Bester BH, Sobuwa S. Utilisation of prehospital intravenous access. South African Medical Journal = Suid-Afrikaanse Tydskrif Vir Geneeskunde. 2014;104(9):615-8.

25. Kuzma K, Sporer KA, Michael GE, Youngblood GM. When are Prehospital Intravenous Catheters Used for Treatment? J Emerg Med. 2009;36(4):357-62.

26. Thomas SH, Shewakramani S. Prehospital trauma analgesia. J Emerg Med. 2008;35(1):47-57.

27. Meek R, Mee MJ, Egerton-Warburton D, Graudins A, Meyer A, Pouryahya P, et al. Randomized Placebo-controlled Trial of Droperidol and Ondansetron for Adult Emergency Department Patients With Nausea. Acad Emerg Med. 2019;26(8):867-77.

28. Coventry LL, Jacob AM, Davies HT, Stoneman L, Keogh S, Jacob ER. Drawing blood from peripheral intravenous cannula compared with venepuncture: A systematic review and meta-analysis. J Adv Nurs. 2019;75(11):2313-39.

29. Egerton-Warburton D, McAllan F, Ramanan R, Lim ZJ, Nagle D, Dendle C, et al. Human factordesigned multimodal intervention reduces the rate of unused peripheral intravenous cannula insertion. Emerg Med Australas. 2019;31(3):372-7.

30. Becerra MB, Shirley D, Safdar N. Prevalence, risk factors, and outcomes of idle intravenous catheters: An integrative review. Am J Infect Control. 2016;44(10):e167-e72.

\section{Figures}




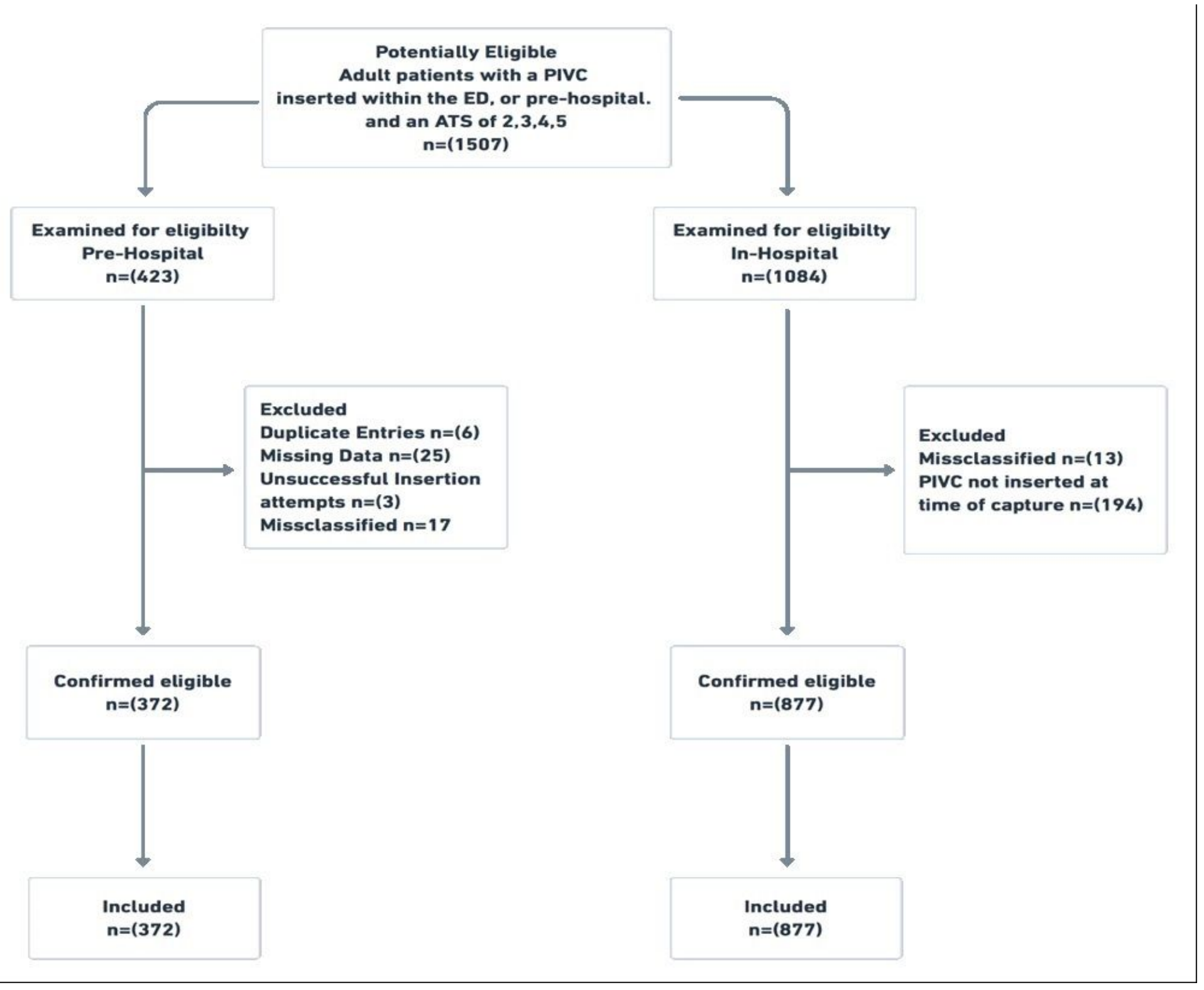

\section{Figure 1}

Patient flow. PIVC: Peripheral intravenous catheter, ED: Emergency Department, ATS: Australasian triage scale. 